\title{
Optimal pest control in agriculture
}

\section{Thomas Christiaans, Thomas Eichner and Rüdiger Pethig}

\author{
University of Siegen \\ Department of Economics \\ Hoelderlinstr. 3 \\ D-57068 Siegen \\ Germany \\ phone: ++49 (0) $2717403164 / 3143$ \\ fax: $++49(0) 2717402732$ \\ e-mail: christiaans@vwl.wiwi.uni-siegen.de \\ e-mail: eichner@vwl.wiwi.uni-siegen.de \\ e-mail: pethig@vwl.wiwi.uni-siegen.de
}

\begin{abstract}
Based on economic methodology we model an ecosystem with two species in predator-prey relationship: mice feed on grain and grain feeds on a resource. With optimizing behavior of individual organisms a short-run ecosystem equilibrium is defined and characterized that depends on the farmer's use of fertilizer and on the mice population which, in turn, is affected by pesticides. In that way, a microfounded agricultural production function is derived. Linking a sequence of short-run ecosystem equilibria yields the growth function of the mice population which is thus derived rather than assumed. In each period the farmer harvests all grain in excess of some given amount of seed. If she maximizes her present-value profits, optimal farming is shown to depend on the prices of pesticide and grain. It is either optimal to use no pesticide or a moderate amount of pesticide or to apply a chattering control. Pest eradication is never optimal. On the other hand, if the farmer takes into account steady state mice populations only, it may be optimal to eradicate mice or to use no or a moderate amount of pesticide depending on prices as well as on the shape of the grain production function which is determined by micro parameters of grain reproduction.
\end{abstract}

JEL classification: Q24, Q57

key words: pesticides, agriculture, predator-prey, chattering pest control 


\section{Introduction}

The economic pest management literature has focused on controlling the growth of a plant and its predator when predators cause major damage by consuming plants (Hall and Norgaard 1973, Feder and Regev 1975). The measures for enhancing crop growth that have received most attention in the literature are pesticides and fertilizers. Despite the rich literature in this field there is an ongoing discussion about specifications and functional forms of agricultural production functions, in particular about the relationship between crop responses to pesticides and fertilizers. For econometric studies of crop responses to the use of pesticides we refer to Babcock, Lichtenberg and Zilberman (1992) and Carrasco-Tauber and Moffitt (1992). For studies of crop responses to the use of fertilizers see Berck and Helfand (1990) or Chambers and Lichtenberg (1996).

In that literature the ecological system is described by dynamic stock-flow relationships which are macro level approaches because they take populations as basic endogenous variables and hence disregard the microstructure of intra-ecosystem transactions. In contrast, the present paper develops a microfoundation of population growth and links this population growth to the application of fertilizers and pesticides. Building on Hannon (1976), Crocker and Tschirhart (1992), Tschirhart (2000, 2002, 2004), Finnoff and Tschirhart (2003a, 2003b) and Eichner and Pethig (2003, 2004) we employ the economic concepts of prices, optimizing behavior and equilibrium to explain the interactions of organisms in predator-prey relationship.

To be more specific, there is a pest species called mice, somewhat allegorically, and a farmer who grows crops (called grain). He harvests the grain at the end of each shortrun period leaving some constant quantity of seeds for the next period. Mice feed on grain and grain feeds on a vital base resource whose supply can be augmented by applying fertilizer. In the short-run period the mice population is constant and the representative individual organisms of mice and grain behave as if they maximize their net-offspring as price takers choosing their prey biomass demand and the supply of own biomass subject to some constraint. The effect of pesticide application to the field is that grain taken up by mice hampers their capacity to reproduce which reduces, ceteribus paribus, the growth rate of mice and hence the future aggregate amount of grain they forage. For any given application of fertilizer and pesticide, the aggregate net offspring of mice being generated at the end of each period constitutes the change in mice population and thus explains the dynamics of the mice population. In other words, the short-run periods serve to determine net offspring along with the flows of prey biomass and resource intake with all populations being fixed. The population dynamics - which are here the dynamics of the mice population 
only because the 'grain population' is kept constant through harvesting - naturally emerge by considering the temporal sequence of short-run periods. Since these population dynamics are specified for any given level of fertilizer and pesticide the links between farming activities and the growth of grain and the mice population are simultaneously established. Thus, our model generates an agricultural production function that accounts for the direct and indirect impact on the growth of grain of fertilization and pesticides, respectively.

After that production function is derived, the paper proceeds to analyze the farmer's profit maximizing pest control. If she maximizes her present-value profits, optimal farming is shown to depend on the prices of pesticide and grain. It is either optimal to use no pesticide or a moderate amount of pesticides or to apply a chattering control which calls for switching between applying no pesticides at all and applying the maximum amount of pesticides. An extinction strategy (which is feasible because the immigration of mice from other patches is ruled out) would be too expensive and is therefore suboptimal. On the other hand, if the farmer takes into account steady state mice populations only, it may be optimal to eradicate mice or to use no or a moderate amount of pesticide depending on prices as well as on the shape of the grain production function which is determined by micro parameters of grain reproduction.

In summary, the paper provides three major contributions. First, it develops a microfoundation of population growth in a predator-prey system with economic-ecological interactions; second, it derives a microfounded parametric agricultural production function and determines its properties; finally, this production function is employed to characterize the farmer's optimal pest control in a dynamic setting.

The paper is organized as follows. In Section 2 we present the short-run model of the ecosystem and derive the population growth functions and the agricultural production function. Section 3 investigates the farmer's dynamic pest control problem, Section 4 studies the farmer's steady-state pest control problem and Section 5 concludes.

\section{Growing crops in an ecosystem with pests}

Consider an ecosystem inhabited by mice in which a farmer grows grain on a field of given size. Mice feed on grain and grain 'feeds on' a resource such as sunlight, minerals or water or other nutrients.

To specify the grain-mice interactions we first consider a short-run period, a point in time, where the populations of grain and mice are constant. Since all organisms of 
a species are assumed to be identical, it suffices to focus on representative organisms. During the short-run period every organism produces net offspring that increases with the amount of prey-biomass intake and declines with the amount of own biomass sacrificed to predators. More specifically, let the net offspring of the representative grain, $g$, and that of the representative mouse, $m$, be given by ${ }^{1}$

$$
\begin{aligned}
g & =x_{r}^{\gamma}\left(\bar{z}_{g}-z_{g}\right)^{1-\gamma}-\omega_{g}, \\
m & =\left[\left(1-c_{m} s\right) x_{g}\right]^{\mu}\left(\bar{z}_{m}-z_{m}\right)^{1-\mu}-\omega_{m} .
\end{aligned}
$$

For $i=r, g$ the demand for prey biomass is $x_{i}$, where $r$ stands for the resource which is called the prey species of grain, for convenience. For $j=g, m$ the supply of own biomass is $\left.z_{j} . \gamma, \mu \in\right] 0,1\left[\right.$ and $c_{m} \geq 0, \bar{z}_{g}, \bar{z}_{m}, \omega_{g}, \omega_{m}>0$ are parameters. $s$ is the amount of pesticide the farmer applies to the field to restrain or prevent mice from feeding on grain. The impact of pesticides on net offspring of pests can be and has been modeled in various different ways (Feder and Regev 1975 and the literature cited therein). In (2) the underlying assumption is that pesticides are directly related to grain consumption of mice and that the net offspring of mice induced by any given grain consumption is the smaller the more pesticides are applied to the field. At each point in time nature makes available the same amount of the resource, $r_{0}>0$. This natural supply is normalized, for convenience, by setting $r_{0}=1$. However, the farmer can augment that supply by applying fertilizer $f$. In that case total supply is $r=r_{0}+f=1+f$. Thus, $f \geq 0$ and $s \in\left[0,1 / c_{m}\right]$ are activities at the farmer's disposal. For the time being these variables are kept constant.

The biomasses of all species are viewed as commodities traded in a system of competitive markets, where 'intake of prey biomass', $x$, translates into 'demand for prey biomass' and 'loss of own biomass', $z$, is interpreted as 'supply of own biomass to predators'. The biomass transfer activities $\left(x_{r}, z_{g}\right)$ and $\left(x_{g}, z_{m}\right)$, the organisms are engaged in, are subject to the constraints

$$
\begin{aligned}
\left(1-\theta_{g} p_{g}\right) e_{g}+p_{g} z_{g} & =p_{r} x_{r} \\
\left(1-\theta_{m} p_{m}\right) e_{m}+p_{m} z_{m} & =p_{g} x_{g}
\end{aligned}
$$

In (3) and (4), $\theta_{i} \geq 0$ and $e_{i}>0$ for $i=g, m$ are constants. $p_{r}, p_{g}$ and $p_{m}$ are nonnegative scarcity indicators called prices, for the resource and the biomass of grain and mice, respectively. All prices are taken as given by the organisms while they are endogenously determined by the competitive mechanism that will be specified further below. For $\theta_{i}=0$ the constraints (3) and (4) look exactly like budget constraints of consumers in

\footnotetext{
${ }^{1}$ For a similar specification of the net offsring functions (without pesticides) and further interpretations of this approach see Christiaans, Eichner and Pethig (2005).
} 
the microeconomic theory of households, where $e_{i}$ would denote exogenous income and $p_{r}$, $p_{g}$ and $p_{m}$ would represent market prices at which the consumer can sell or purchase the respective products. ${ }^{2}$ However, since the ecosystem model is not monetized (a property which it actually shares with the neoclassical model of the economy!) prices and incomes are denominated in virtual units of account. To see that the isomorphism between consumers and organisms of nonhuman species makes sense, observe that (in case of $\theta_{i}=0$ ) the 'exogenous income' $e_{i}$ reflects an organism's predation power in the sense that for any given price of prey biomass this income determines the amount of prey biomass, $e_{g} / p_{r}$ or $e_{m} / p_{g}$, the organism is capable to purchase without being forced to sacrifice own biomass. However, purchasing prey biomass beyond that amount requires to sacrifice own biomass. This implication of the constraints (3) and (4) readily reflects what ecologists refer to as the organism's predation risk.

In case of a positive (and constant) parameter $\theta_{i}$, organism $i$ 's 'exogenous income' is still $e_{i}$, if and only if $p_{i}=0$. Otherwise it is $\left(1-\theta_{i} p_{i}\right) e_{i}<e_{i}$, and it becomes even negative, if $p_{i}$ exceeds some positive threshold value. The idea behind that hypothesis is that the scarcer is the biomass of the organism under consideration, that is, the higher is the predation pressure on that organism and hence the higher is $p_{i}$, the more it is forced to yield own biomass to its predators possibly to such an extent that it is not compensated for its sacrifice by an intake of its own prey 'in exchange'. The size of $\theta_{i}$ will turn out to have an important impact on results in Section 4.

Grain organisms maximize (1) subject to (3) with respect to $x_{r}$ and $z_{g}$ which yields their demand for the basic resource and their supply of own biomass,

$$
x_{r}=\frac{p_{g}}{p_{r}} \gamma \bar{z}_{g}^{\theta}+\gamma \frac{e_{g}}{p_{r}}, \quad z_{g}=e_{g} \theta_{g}+\gamma \bar{z}_{g}^{\theta}-(1-\gamma) \frac{e_{g}}{p_{g}}, \quad\left(p_{r}>0, p_{g}>0\right)
$$

where $\bar{z}_{g}^{\theta}:=\bar{z}_{g}-e_{g} \theta_{g}$. Maximization of (2) subject to (4) with respect to $x_{g}$ and $z_{m}$ gives rise to the mice's demand for grain and their supply of own biomass,

$$
\begin{array}{lll}
x_{g}=\frac{p_{m}}{p_{g}} \mu \bar{z}_{m}^{\theta}+\mu \frac{e_{m}}{p_{g}}, & z_{m}=e_{m} \theta_{m}+\mu \bar{z}_{m}^{\theta}-(1-\mu) \frac{e_{m}}{p_{m}}, & \left(p_{g}>0, p_{m}>0\right), \\
x_{g}=\frac{e_{m}}{p_{g}} & z_{m}=0, & \left(p_{g}>0, p_{m}=0\right),
\end{array}
$$

where $\bar{z}_{m}^{\theta}:=\bar{z}_{m}-e_{m} \theta_{m}$.

It is useful to briefly check how grain organism $i$ adjusts its demand and supply to

\footnotetext{
${ }^{2}$ The counterpart of 'supply of own biomass' in the economic consumer theory would be 'supply of labor'.
} 
parametric changes in prices and in its constraint parameter $\theta_{i}$. (5) readily yields

$$
\begin{aligned}
& \frac{\partial z_{g}}{\partial \theta_{g}}>0, \frac{\partial z_{g}}{\partial p_{r}}=0, \frac{\partial z_{g}}{\partial p_{g}}>0, \frac{\partial x_{r}}{\partial \theta_{g}}<0, \frac{\partial x_{r}}{\partial p_{r}}<0, \quad \text { and } \\
& \frac{\partial x_{r}}{\partial p_{g}} \gtreqless 0 \quad \Longleftrightarrow \quad \bar{z}_{g}^{\theta}:=\bar{z}_{g}-e_{g} \theta_{g} \gtreqless 0 .
\end{aligned}
$$

According to (7) a grain organism increases its supply of own biomass as well as its demand for prey biomass as a response to an increase in either $p_{g}$ or $\theta_{g}$, if and only if $\theta_{g}<\tilde{\theta}_{g}:=$ $\bar{z}_{g} / e_{g}>0$. In that case rising scarcity of grain $\left(\mathrm{d} p_{g}>0\right)$ has two opposing effects on grain net offspring. That offspring is reduced by the increased loss of own biomass $\left(\partial z_{g} / \partial p_{g}>0\right)$ but it is fostered by an increase in prey biomass intake $\left(\partial x_{r} / \partial p_{g}>0\right)$. The response of grain to growing predation pressure is then to build up more pressure on its own prey. On the other hand, if $\theta_{g}>\tilde{\theta}_{g}$, an increase in scarcity $\left(\mathrm{d} p_{g}>0\right)$ reduces net offspring inadvertantly and reduces the pressure of mice on the resource. In this sense grain can be called a weak or a strong species depending on whether $\theta_{g}>\tilde{\theta}_{g}$ or $\theta_{g}<\tilde{\theta}_{g}$.

A short-run ecosystem equilibrium is constituted by a vector of transactions $\left(x_{r}, z_{g}\right.$, $\left.x_{g}, z_{m}\right)$ and prices $\left(p_{r}, p_{g}, p_{m}\right)$ such that $(5)$ and (6) satisfy the market clearing conditions

$$
\begin{aligned}
n_{g} x_{r} & =1+r=f, \\
n_{m} x_{g} & =n_{g} z_{g}, \\
n_{b} x_{m} & =n_{m} z_{m} .
\end{aligned}
$$

Equation (8c) demands equilibrium on the market for mice biomass, where $n_{b} x_{m}$ denotes total demand of mice biomass by all members, $n_{b}$, of some species that preys on mice. However, in the food chain studied here mice are the top predators by assumption and therefore there is no species, developing a demand for mice biomass. Yet it is interesting to see with the help of $(8 \mathrm{c})$ how the nonexistence of a market for mice biomass is derived as a natural implication of the formal model. Let $n_{b}$ be the population of a species, say buzzards, that would prey on mice if it would inhabit the ecosystem. Since it doesn't, we set $n_{b}=0$ which implies $x_{m}=0$. For that reason (8c) is satisfied if and only if $z_{m}=0$. Sufficient for $z_{m}=0$ is $p_{m}=0$ which is just another way of saying that a market for mice biomass doesn't exist.

The next step is to calculate equilibrium prices and quantities. To facilitate notation, it is convenient to introduce at this point already the assumption that in every period the farmer harvests all grain in excess of some given grain population (transferred as seed to the next period) which we normalize to be $n_{g}=\bar{n}_{g}$. Hence we set $n_{g}=\bar{n}_{g}$ in (8a) and $(8 \mathrm{~b})$ and insert the demand and supply functions (5) and (6b) into the market clearing 
conditions (8a) and (8b) to obtain the equilibrium prices

$$
p_{r}=\frac{\gamma\left(\bar{n}_{g} e_{g} \bar{z}_{g}+n_{m} e_{m} \bar{z}_{g}^{\theta}\right)}{r \hat{z}_{g}}, \quad p_{g}=\frac{\bar{n}_{g} e_{g}(1-\gamma)+n_{m} e_{m}}{\hat{z}_{g}}, \quad p_{m}=0,
$$

where $\hat{z}_{g}:=e_{g} \theta_{g}+\gamma \bar{z}_{g}^{\theta}$. In view of (8), (5) and (6b), the equilibrium transfers are

$$
\begin{array}{rlrl}
x_{r} & =\frac{r}{\bar{n}_{g}}, & z_{g} & =\frac{n_{m} e_{m} \hat{z}_{g}}{\bar{n}_{g} e_{g}(1-\gamma)+n_{m} e_{m}}, \\
x_{g}=\frac{\bar{n}_{g}}{n_{m}} \cdot \frac{n_{m} e_{m} \hat{z}_{g}}{\bar{n}_{g} e_{g}(1-\gamma)+n_{m} e_{m}}, & z_{m} & =0 .
\end{array}
$$

In (9) and (10) we have fully specified the equilibrium allocation of the short-run ecosystem model. To see the rich implications of that model and its predictive power we summarize some selected comparative statics in Table 1. The first row of that Table introduces small parameter changes (exogenous shocks) and the first column lists the equilibrium adjustments of all endogenous variables to these shocks. ${ }^{3}$

\begin{tabular}{|c||c|c|c|c|c|c|c|}
\hline & $\partial e_{g}$ & $\partial e_{m}$ & $\partial \bar{z}_{g}$ & $\partial r$ & $\partial \bar{n}_{g}$ & $\partial n_{m}$ & $\partial \theta_{g}$ \\
\hline \hline$\partial p_{r}$ & $?,+^{a}$ & + & + & - & + & + & - \\
\hline$\partial p_{g}$ & $?,+$ & + & - & 0 & + & + & - \\
\hline$\partial x_{r}$ & 0 & 0 & 0 & + & - & 0 & 0 \\
\hline$\partial x_{g}$ & $?,-$ & + & + & 0 & + & - & + \\
\hline$\partial z_{g}$ & $?,-$ & + & + & 0 & - & + & + \\
\hline
\end{tabular}

${ }^{a}$ The second sign in this row is obtained in the special case $\theta_{g}=0$.

Table 1: The comparative statics of the short-run ecosystem equilibrium

Leaving out some detail and special features of Table 1 the principal results of the exercise in comparative statics are:

(i) If the 'exogenous income' $e_{i}$ of organism $i=g, m$ increases, then

- all prices increase,

- organism $i$ benefits either through a reduction of own biomass loss (in case of $i=g$ ) or through an increase in biomass intake (in case of $i=m$ ).

(ii) If the population $n_{i}$ of species $i=g, m$ increases, then

\footnotetext{
${ }^{3}$ For example, the box associated to the fifth column and fourth row contains the information $\partial x_{r} / \partial r>0$, i.e. an exogenous increase in the supply of the resource induces a rise in consumption of the resource by each mouse.
} 
- all prices increase,

- organism $i$ reduces its market transactions, while organism $j \neq i$ expands its market transactions.

(iii) If the parameter $\theta_{g}$ increases, then

- all prices decrease,

- all organisms expand their market transactions.

The next step is to link consecutive short-run periods to focus on the growth (or decline) of species over time. This population update is done by simply inserting (10a) in (1) and (10b) in (2):

$$
\begin{aligned}
& \frac{\dot{n}_{g}}{\bar{n}_{g}}=g=\left(\frac{1+f}{\bar{n}_{g}}\right)^{\gamma}\left[\frac{(1-\gamma)\left(\bar{n}_{g} e_{g} \bar{z}_{g}+n_{m} e_{m} \bar{z}_{g}^{\theta}\right)}{\bar{n}_{g} e_{g}(1-\gamma)+n_{m} e_{m}}\right]^{1-\gamma}-\omega_{g}=: G\left(f, n_{m}\right), \\
& \frac{\dot{n}_{m}}{n_{m}}=m=\left(1-c_{m} s\right)^{\mu}\left[\frac{\bar{n}_{g} e_{m} \hat{z}_{g}}{\bar{n}_{g} e_{g}(1-\gamma)+n_{m} e_{m}}\right]^{\mu} \bar{z}_{m}^{1-\mu}-\omega_{m} .
\end{aligned}
$$

It should be emphasized that the differential equations (11) are solely built on the information that - and do not use any other information than that which - has been introduced in the model of the short-run period to determine the behavior and the transactions of individual organisms. In that sense the population growth functions (11) are microfounded. They are derived from more basic concepts whereas conventional models of population ecology start out with population growth functions as their basic concepts.

We now wish to characterize $\dot{n}_{m}$ as a function of $n_{m}$. Define [.] $:=\bar{n}_{g} e_{g}(1-\gamma)+n_{m} e_{m}$, for convenience of notation, and differentiate (11b):

$$
\begin{aligned}
\frac{\mathrm{d} \dot{n}_{m}}{\mathrm{~d} n_{m}} & =\left(1-c_{m} s\right)^{\mu}\left(\bar{n}_{g} e_{m} \hat{z}_{g}\right)^{\mu}\left\{[\cdot]^{\mu}-n_{m} e_{m} \mu[\cdot]^{-\mu-1}\right\}-\omega_{m} \\
\frac{\mathrm{d}^{2} \dot{n}_{m}}{\mathrm{~d} n_{m}^{2}} & =-\left(1-c_{m} s\right)^{\mu}\left(\bar{n}_{g} e_{m} \hat{z}_{g}\right)^{\mu} \mu e_{m} \bar{z}_{m}^{1-\mu}[\cdot]^{-\mu-1}\left\{2-\frac{n_{m} e_{m}(1+\mu)}{[\cdot]}\right\}<0 .
\end{aligned}
$$

It is obvious from (11b) that $\dot{n}_{m}=0$ is satisfied for $n_{m}=0$ as well as for

$$
n_{m}^{s s}=N^{m}(s):=\left(1-c_{m} s\right) \bar{n}_{g} \hat{z}_{g} \frac{\bar{z}_{m}^{\frac{1-\mu}{\mu}}}{\omega_{m}^{\frac{1}{\mu}}}-\frac{(1-\gamma) \bar{n}_{g} e_{g}}{e_{m}} .
$$

Note also that $\frac{\mathrm{d} \dot{n}_{m}}{\mathrm{~d} n_{m}}>0$ at $n_{m}=0$, if and only if $\left(1-c_{m} s\right)^{\mu}\left(\bar{n}_{g} e_{g} \hat{z}_{g}\right)^{\mu}\left(\bar{n}_{g} e_{g}(1-\gamma) z_{g}\right)^{\mu}<\omega_{m}$ which will be assumed in what follows. When combined with (13) and the observation that $\dot{n}_{m}=0$ at $n_{m}=0$ and at $n_{m}=n_{m}^{s s}$ this assumption proves that $\dot{n}_{m}$ is an inverse u-shaped 
function of $n_{m}$ with $n_{m}^{s s}$, representing the carrying capacity of mice. ${ }^{4}$ Hence we established that for any constant application of pesticide, the mice population exhibits logistic growth.

$G\left(f, n_{m}\right)$ as defined in (11a) is the net offspring of the representative grain organism which is determined by fertilization and by the mice population. The total grain available at the end of the period under consideration is $\bar{n}_{g} G\left(f, n_{m}\right)$ which therefore represents the farmer's short-run or rather instantaneous production function. In the literature on agricultural production the notion and study of pesticide productivity plays a prominent role (e.g. Carrasco-Tauber and Moffitt (1992), Babcock et al. (1992)). It is therefore all the more intriguing that in our model the grain production function is independent of pesticide use, $s$. This property $G_{s}=0$ is a consequence of our assumption on the impact of pesticides on mice net offspring in (2). Due to this modeling approach all biomass demands and supplies in (5) and (6) are independent of $s$ and so is also the equilibrium allocation (9) and (10). However, the observation that $G$ is independent of $s$ does not mean that pesticides do not matter. It rather means that the use of pesticides affects the production of grain only through its impact on the mice population, $n_{m}$. That population is constant by assumption in the short-run period but it changes in time according to the growth function (11b) which clearly depends on $s$.

To sum up, in the present model the use of pesticide affects short-run grain production not directly $\left(G_{s}=0\right)$ but only indirectly through its impact on the mice population dynamics. Taking a closer look at that production function we characterize the function $G$ as follows:

\section{Proposition 1 .}

(i) $G\left(f, n_{m}\right)$ is strictly increasing and strictly concave in $f$.

(ii) (a) $G\left(f, n_{m}\right)$ is strictly decreasing in $n_{m}$.

(b) $G\left(f, n_{m}\right)$ is strictly convex (concave) in $n_{m}$ if and only if $\theta_{g}<(>) \hat{\theta}_{g}$ $:=\frac{\bar{z}_{g} \bar{n}_{g}(2-\gamma)}{2 n_{m} e_{m}+\bar{n}_{g} e_{g}(1-\gamma) \gamma}+\frac{\bar{z}_{g}}{e_{g}}$.

\footnotetext{
${ }^{4}$ As defined in (14), the carrying capacity depends on - and is hence explained by - the characteristics (micro parameters) of both grain and mice in a complex way. That gives rise to a variety of interesting testable hypotheses which will not be elaborated here, however.
} 
Proof: Differentiation of $G$ with respect to $n_{m}$ yields after some rearrangement of terms

$$
\begin{aligned}
G_{n_{m}}= & -\frac{\left(\frac{1+f}{\bar{n}_{g}}\right)^{\gamma}(1-\gamma)^{2} e_{g} e_{m}\left[\bar{z}_{g}-(1-\gamma) \bar{z}_{g}^{\theta}\right]\left[\frac{(1-\gamma)\left(\bar{n}_{g} e_{g} \bar{z}_{g}+n_{m} e_{m} \bar{z}_{g}^{\theta}\right)}{\bar{n}_{g} e_{g}(1-\gamma)+n_{m} e_{m}}\right]^{-\gamma}}{\left[\bar{n}_{g} e_{g}(1-\gamma)+n_{m} e_{m}\right]^{2}}, \\
G_{n_{m} n_{m}}= & \frac{\left(\frac{1+f}{\bar{n}_{g}}\right)^{\gamma} \bar{n}_{g}(1-\gamma)^{3} e_{g} e_{m}^{2}\left[\bar{z}_{g}-(1-\gamma) \bar{z}_{g}^{\theta}\right]\left[\frac{(1-\gamma)\left(\bar{n}_{g} e_{g} \bar{z}_{g}+n_{m} e_{m} \bar{z}_{g}^{\theta}\right)}{\bar{n}_{g} e_{g}(1-\gamma)+n_{m} e_{m}}\right]^{-\gamma-1}}{\left[\bar{n}_{g} e_{g}(1-\gamma)+n_{m} e_{m}\right]^{4}} \\
& \cdot\left[\bar{z}_{g} \bar{n}_{g} e_{g}(2-\gamma)+\bar{z}_{g}^{\theta}\left(2 n_{m} e_{m}+\bar{n}_{g} e_{g}(1-\gamma) \gamma\right)\right] .
\end{aligned}
$$

Observe that $G_{n_{m}}<0$ due to $\bar{z}_{g}-(1-\gamma) \bar{z}_{g}^{\theta}=\gamma \bar{z}_{g}+(1-\gamma) e_{g} \theta_{g}=: \hat{z}_{g}>0$. In addition, we find

$$
G_{n_{m} n_{m}} \lesseqgtr 0 \quad \Longleftrightarrow \quad \bar{z}_{g} \bar{n}_{g} e_{g}(2-\gamma)+\bar{z}_{g}^{\theta}\left(2 n_{m} e_{m}+\bar{n}_{g} e_{g}(1-\gamma) \gamma\right) \lesseqgtr 0 .
$$

Using $\bar{z}_{g}^{\theta}=\bar{z}_{g}-e_{g} \theta_{g}$, the condition on the right side of (17) is equivalent to $\frac{\bar{z}_{g} \bar{n}_{g}(2-\gamma)}{2 n_{m} e_{m}+\bar{n}_{g} e_{g}(1-\gamma) \gamma}+$ $\frac{\bar{z}_{g}}{e_{g}} \lesseqgtr \theta_{g}$.

The negative impact of the mice population on the growth of grain is as expected $\left(G_{n_{m}}<0\right.$ in Proposition 1). Not so clear is, however, whether the mice population hampers the growth of grain more than (convex) or less than (concave) proportional. Ceteribus paribus, the curvature of $G$ depends on the parameter $\theta_{g}$. Sufficient for the convexity of $G$ is $\theta_{g}<\tilde{\theta}_{g}:=\bar{z}_{g} / e_{g}$ which characterized mice as a strong species (as noted above). The weaker the mice species is the more likely it is that $G$ becomes strictly concave in $n_{m}$.

\section{Optimal dynamic pest control}

Consider now the farmer's grain growing activity. She faces the instantaneous production function $\bar{n}_{g} G\left(f, n_{m}\right)$ with $G(\cdot)$ from (11a). Owing to our simplifying assumption that in each period the farmer retains the amount $\bar{n}_{g}$ of grain to be transferred as seed to the next period, the grain for sale in the market is $\bar{n}_{g} G\left(f, n_{m}\right)-\bar{n}_{g}$ (which will be assumed to be nonnegative). The farmer also takes into account the equation of motion (11b), the constant and time-invariant prices for grain, $\pi_{g}$, for fertilizer, $\pi_{f}$, for pesticides, $\pi_{s}$, and she solves

$$
\begin{array}{r}
\max _{f_{t}, s_{t}} \int_{0}^{\infty} e^{-\delta t}\left[\pi_{g}\left(\bar{n}_{g} G\left(f_{t}, n_{m t}\right)-\bar{n}_{g}\right)-\pi_{f} f_{t}-\pi_{s} s_{t}\right] \mathrm{d} t \\
\text { subject to } 0 \leq s_{t} \leq 1 / c_{m}, \quad n_{m}(0)=n_{m 0} \in\left[0, \bar{n}_{m}\right], \\
\dot{n}_{m t}=n_{m t}\left\{\left(1-c_{m} s\right)^{\mu}\left[\frac{\bar{n}_{g} e_{m} \hat{z}_{g}}{\bar{n}_{g} e_{g}(1-\gamma)+n_{m} e_{m}}\right]^{\mu} \bar{z}_{m}^{1-\mu}-\omega_{m}\right\},
\end{array}
$$


where $n_{m 0} \in\left[0, \bar{n}_{m}\right]$ and where $\bar{n}_{m}=N^{m}(0)$ denotes the maximum value of $n_{m}$ with the function $N^{m}$ given by (14). The current-value Hamiltonian

$$
\begin{aligned}
H=\pi_{g}\left[\bar{n}_{g} G\left(f_{t}, n_{m t}\right)-\bar{n}_{g}\right]- & \pi_{f} f_{t}-\pi_{s} s_{t} \\
& +\lambda_{t} n_{m t}\left\{\left(1-c_{m} s\right)^{\mu}\left[\frac{\bar{n}_{g} e_{m} \hat{z}_{g}}{\bar{n}_{g} e_{g}(1-\gamma)+n_{m} e_{m}}\right]^{\mu} \bar{z}_{m}^{1-\mu}-\omega_{m}\right\}
\end{aligned}
$$

is strictly concave in $f_{t}$ if $\pi_{g}>0$ because $G$ is strictly concave in $f_{t}$. As $\left(1-c_{m} s_{t}\right)^{\mu}$ is strictly concave in $s_{t}$, the Hamiltonian is strictly concave (strictly convex) in $s_{t}$ if $\lambda_{t}>0$ $\left(\lambda_{t}<0\right)$. Since $H$ can be written $H=H^{1}\left(f_{t}\right)+H^{2}\left(s_{t}\right)$, that function is additively separable in $f_{t}$ and $s_{t}$. Hence maximization of $H$ requires to maximize $H^{1}$ with respect to $f_{t}$ and $H^{2}$ with respect to $s_{t}$ without the need to consider cross partial derivatives. Maximization of the Hamiltonian is thus possible for an interior value of $f_{t}$, which will be assumed, while a boundary solution with respect to $s_{t}$ will emerge if $\lambda_{t}<0$.

Optimum Conditions In order to simplify notation, the time index $t$ will be suppressed. Necessary optimum conditions are

$$
\begin{aligned}
H_{f} & =\pi_{g} \bar{n}_{g} G_{f}\left(f, n_{m}\right)-\pi_{f}=0, \\
& \max _{s} H\left(n_{m}, f, s, \lambda\right), \\
\dot{\lambda} & =\delta \lambda-\pi_{g} \bar{n}_{g} G_{n_{m}}\left(f, n_{m}\right)-\lambda\left\{\left(1-c_{m} s\right)^{\mu}\left[\frac{\bar{n}_{g} e_{m} \hat{z}_{g}}{\bar{n}_{g} e_{g}(1-\gamma)+n_{m} e_{m}}\right]^{\mu} \bar{z}_{m}^{1-\mu}-\omega_{m}\right\} \\
& +\lambda \frac{n_{m}\left(1-c_{m} s\right)^{\mu} \mu e_{m} \bar{z}_{m}^{1-\mu}}{\bar{n}_{g} e_{g}(1-\gamma)+n_{m} e_{m}}\left[\frac{\bar{n}_{g} e_{m} \hat{z}_{g}}{\bar{n}_{g} e_{g}(1-\gamma)+n_{m} e_{m}}\right]^{\mu} .
\end{aligned}
$$

The derivative of $H$ with respect to $s$ is

$$
H_{s}=-\pi_{s}-\lambda n_{m} c_{m} \mu\left(1-c_{m} s\right)^{\mu-1}\left[\frac{\bar{n}_{g} e_{m} \hat{z}_{g}}{\bar{n}_{g} e_{g}(1-\gamma)+n_{m} e_{m}}\right]^{\mu} \bar{z}_{m}^{1-\mu}
$$

Convexity of the Hamiltonian We begin by proving that $\lambda<0$. Suppose on the contrary that $\lambda \geq 0$ at some time $t_{1}$. If $\lambda=0$, equation (21) implies that $\dot{\lambda}>0$ (as $\left.G_{n_{m}}<0\right)$ and therefore $\lambda>0$ for all $t>t_{1}$. Thus, we may as well suppose that $\lambda>0$ right from the beginning. This implies that $H_{s}<0$ for all $s \in\left[0,1 / c_{m}\right]$; hence, $s=0$ maximizes the Hamiltonian and $n_{m}$ converges to $n_{m}^{s s}=N_{m}(0)>0$ according to (14). Owing to (21), $\lambda$ asymptotically follows the differential equation

$$
\dot{\lambda}=\delta \lambda-\pi_{g} \bar{n}_{g} \underbrace{G_{n_{m}}\left(f, n_{m}^{s s}\right)}_{=: C_{1}}+\lambda \underbrace{\frac{n_{m}^{s s} \mu e_{m} \bar{z}_{m}^{1-\mu}}{\bar{n}_{g} e_{g}(1-\gamma)+n_{m} e_{m}}\left[\frac{\bar{n}_{g} e_{m} \hat{z}_{g}}{\bar{n}_{g} e_{g}(1-\gamma)+n_{m} e_{m}}\right]^{\mu}}_{=: C_{2}},
$$


where $C_{1}<0$ and $C_{2}>0$ are constant. As we are in a steady state when $\dot{n}_{m}=0$ and $s$ is constant, the optimum conditions also require $\dot{\lambda}=0$. This implies

$$
\lambda=\frac{\pi_{g} \bar{n}_{g} G_{n_{m}}\left(f, n_{m}^{s s}\right)}{\delta+C_{2}}<0,
$$

a contradiction. Thus, $\lambda(t)<0$ for all $t$ and the Hamiltonian is strictly convex in $s$ due to our assumption $\mu \in] 0,1[$.

Linearized Problem Before we further explore the convex control problem stated above we first analyze an easier-to-handle, linearized version in which $\mu \in] 0,1[$ is replaced by $\mu=1 .^{5}$ Substituting $\left(1-c_{m} s\right)$ for $\left(1-c_{m} s\right)^{\mu}$ in $(11 \mathrm{~b})$ yields the current-value Hamiltonian

$$
\begin{aligned}
H & =\pi_{g}\left(\bar{n}_{g} G\left(f, n_{m}\right)-\bar{n}_{g}\right)-\pi_{f} f-\pi_{s} s \\
& +\lambda_{t} n_{m}\left\{\left(1-c_{m} s\right)\left[\frac{\bar{n}_{g} e_{m} \hat{z}_{g}}{\bar{n}_{g} e_{g}(1-\gamma)+n_{m} e_{m}}\right]^{\mu} \bar{z}_{m}^{1-\mu}-\omega_{m}\right\},
\end{aligned}
$$

which is strictly concave in $f$ and linear in $s$. Note that at the boundaries $s=0$ and $s=1 / c_{m}$ the solutions of the convex and the linearized problem coincide. For simplicity, we assume an interior solution with respect to $f$. According to (19), it is then possible to insert the optimum value $f^{*}$ as a function of $\pi_{f} / \pi_{g}$ and $n_{m}$ into $G$, yielding a problem that is pre-optimized with respect to $f$. The pre-optimized problem contains just one control variable, $s$.

Defining

$$
\begin{gathered}
A\left(n_{m}\right):=\pi_{g}\left(\bar{n}_{g} G\left(f^{*}, n_{m}\right)-\bar{n}_{g}\right)-\pi_{f} f^{*}, B\left(n_{m}\right):=-\pi_{s}, \Phi:=s \\
a\left(n_{m}\right):=n_{m}\left[\frac{\bar{n}_{g} e_{m} \hat{z}_{g}}{\bar{n}_{g} e_{g}(1-\gamma)+n_{m} e_{m}}\right]^{\mu} \bar{z}_{m}^{1-\mu}-n_{m} \omega_{m}, \\
b\left(n_{m}\right):=-c_{m} n_{m}\left[\frac{\bar{n}_{g} e_{m} \hat{z}_{g}}{\bar{n}_{g} e_{g}(1-\gamma)+n_{m} e_{m}}\right]^{\mu} \bar{z}_{m}^{1-\mu}
\end{gathered}
$$

and applying the transformations

$$
M\left(n_{m}\right):=A\left(n_{m}\right)-a\left(n_{m}\right) B\left(n_{m}\right) / b\left(n_{m}\right), N\left(n_{m}\right):=B\left(n_{m}\right) / b\left(n_{m}\right)
$$

puts the problem into the form

$$
\begin{array}{r}
\max _{s} \int_{0}^{\infty} e^{-\delta t}\left[M\left(n_{m}\right)+N\left(n_{m}\right) \dot{n}_{m}\right] \mathrm{d} t \\
\text { subject to } n_{m}(0)=n_{m 0} \in\left[0, \bar{n}_{m}\right], \\
\dot{n}_{m} \in\left\{a\left(n_{m}\right)+b\left(n_{m}\right) s \mid s \in\left[0,1 / c_{m}\right]\right\}, \quad n_{m} \in\left[0, \bar{n}_{m}\right] .
\end{array}
$$

\footnotetext{
${ }^{5}$ Linear crop production functions with limiting inputs are widely used and estimated in the agricultural literature, e.g. in Lanzer and Paris (1981) and in Grimm et al. (1987). These so-called linear response and plateau or von Liebig models refer to a plant's response to nutrients, however, while in our approach $\mu=1$ linearizes the net offspring function of the representative mouse.
} 
This problem can be handled using Proposition 2 stated below, which relies on Theorem 3.2 and Note 3.2 in Feichtinger und Hartl (1986, pp. 68-73) adjusted for the present purpose. That Proposition 2 employs the notion of the fastest possible adjustment path (FPAP) with the following intuitive meaning: A FPAP to another given path $n_{m}^{*}(t)$ is a path $n_{m}^{* *}(t)$ that at any instant $t$ comes closer to $n_{m}^{*}(t)$ than any other path $n_{m}^{* * *}(t)$.

\section{Proposition 2 .}

(i) Let $q$ be a natural number and let $\hat{n}_{m 1}, \ldots, \hat{n}_{m q}, \tilde{n}_{m 1}, \ldots, \tilde{n}_{m q-1}$ be admissible solutions of 6

$$
\delta N\left(n_{m}\right)+M^{\prime}\left(n_{m}\right)=0,
$$

satisfying $\hat{n}_{m 1}<\tilde{n}_{m 1}<\hat{n}_{m 2}<\tilde{n}_{m 2}<\ldots<\tilde{n}_{m q-1}<\hat{n}_{m q}$ and

$$
\delta N\left(n_{m}\right)+M^{\prime}\left(n_{m}\right) \begin{cases}>0 & \text { if } \quad n_{m} \in\left(\tilde{n}_{m i-1}, \hat{n}_{m i}\right) \quad \text { or } \quad 0 \leq n_{m}<\hat{n}_{m 1} \\ <0 & \text { if } \quad n_{m} \in\left(\hat{n}_{m i}, \tilde{n}_{m i}\right) \quad \text { or } \quad \hat{n}_{m q}<n_{m} \leq \bar{n}_{m}\end{cases}
$$

Then, if it exists, the FPAP to one of the equilibria $\hat{n}_{m 1}, \ldots, \hat{n}_{m q}$ is the solution of $(24) \cdot{ }^{7}$

(ii) If there is an additional solution $\tilde{n}_{m q}>\hat{n}_{m q}\left(\tilde{n}_{m 0}<\hat{n}_{m 1}\right.$, respectively) of

$$
\delta N\left(n_{m}\right)+M^{\prime}\left(n_{m}\right)=0,
$$

$n_{m}$ is bounded from above by $\bar{n}_{m} \leq \infty$ (from below by 0 , respectively) and $\delta N\left(\bar{n}_{m}\right)+$ $M^{\prime}\left(\bar{n}_{m}\right)>0\left(\delta N(0)+M^{\prime}(0)<0\right.$, respectively $)$, where $\bar{n}_{m}>\hat{n}_{m q}\left(0<\tilde{n}_{m 0}\right.$, respectively), the FPAP to one of the equilibria $\hat{n}_{m 1}, \ldots, \hat{n}_{m q}$ or to $\bar{n}_{m}(0$, resp.) is the solution of (24).

(iii) If $\delta N\left(n_{m}\right)+M^{\prime}\left(n_{m}\right)>0\left(\delta N\left(n_{m}\right)+M^{\prime}\left(n_{m}\right)<0\right.$, respectively) for all $n_{m} \in\left[0, \bar{n}_{m}\right]$, the FPAP to $\bar{n}_{m}$ (0, respectively) is the solution of (24).

For the problem at hand, the functions $M$ and $N$ are given by

$$
\begin{gathered}
M\left(n_{m}\right)=\pi_{g}\left[\bar{n}_{g} G\left(f^{*}, n_{m}\right)-\bar{n}_{g}\right]-\pi_{f} f^{*}+\pi_{s}\left\{\left[\frac{\bar{n}_{g} e_{m} \hat{z}_{g}}{\bar{n}_{g} e_{g}(1-\gamma)+n_{m} e_{m}}\right]^{\mu} \bar{z}_{m}^{1-\mu}-\frac{1}{c_{m}}\right\}, \\
N\left(n_{m}\right)=\frac{\pi_{s}}{c_{m} n_{m} \bar{z}_{m}^{1-\mu}}\left[\frac{\bar{n}_{g} e_{m} \hat{z}_{g}}{\bar{n}_{g} e_{g}(1-\gamma)+n_{m} e_{m}}\right]^{\mu} .
\end{gathered}
$$

\footnotetext{
${ }^{6} M^{\prime}$ denotes the derivative of $M$ with respect to $n_{m}$.

${ }^{7}$ In addition, the condition $\lim _{t \rightarrow \infty} e^{-\delta t} \int_{n_{m}(t)}^{\hat{n}_{m}} N(x) \mathrm{d} x \geq 0$ must be satisfied for any optimum singular level $\hat{n}_{m}$ and any admissible solution $n_{m}(t)$. Since for the present problem $N(x)$ is always positive and bounded from above except for $x=0$, this condition is always met.
} 
The condition $\delta N\left(n_{m}\right)+M^{\prime}\left(n_{m}\right)=0$ is equivalent to

$$
\delta N\left(n_{m}\right)+\pi_{g}\left[\bar{n}_{g} G_{n_{m}}\left(f^{*}, n_{m}\right)-\bar{n}_{g}\right]+\frac{\pi_{s} \mu e_{m}}{\bar{n}_{g} e_{g}(1-\gamma)+n_{m} e_{m}}\left[\frac{\bar{n}_{g} e_{m} \hat{z}_{g}}{\bar{n}_{g} e_{g}(1-\gamma)+n_{m} e_{m}}\right]^{\mu}=0 .
$$

For $G_{n_{m}}\left(f^{*}, n_{m}\right)$ we refer to equation (15).

Unfortunately, analytical results are hardly obtainable except for the result $\lim _{n_{m} \rightarrow 0}$ $\delta N\left(n_{m}\right)+M^{\prime}\left(n_{m}\right)=+\infty$. For that reason, we present in the following a numerical example based on the parameter values: $\delta=0.05, e_{g}=e_{m}=1, \gamma=\mu=0.5, \bar{n}_{g}=1, \theta_{g}=\theta_{m}=0$, $\omega_{g}=\omega_{m}=50, \bar{z}_{g}=\bar{z}_{m}=10000, \pi_{g} / \pi_{f}=0.5$. First observe that from (19) we infer that it is optimal to use no fertilizers at all, $f^{*}=0$. The function $\delta N\left(n_{m}\right)+M^{\prime}\left(n_{m}\right)$ is presented in ${ }^{8}$ figures 1-3 for different price ratios $\pi_{g} / \pi_{s}$.

Case I: If $\pi_{g} / \pi_{s}$ is sufficiently close to zero, it is optimal to apply no pesticides at all. If $\pi_{g} / \pi_{s}>0$ but very small, $\delta N\left(n_{m}\right)+M^{\prime}\left(n_{m}\right)$ can be shown to be positive for all $n_{m} \in$ $\left[0, \bar{n}_{m}\right]$, and the shape of the $\delta N\left(n_{m}\right)+M^{\prime}\left(n_{m}\right)$ curve is as depicted in Figure 1. In that case the FPAP to $\bar{n}_{m}$ is optimal according to part (iii) of Theorem 1 . Recall that $\bar{n}_{m}=N^{m}(0)$ by definition, where $N^{m}(0)$ represents the carrying capacity of mice for $s=0$ according to (14). Hence both in the short run and in the long run it is optimal to apply no pesticides at all.

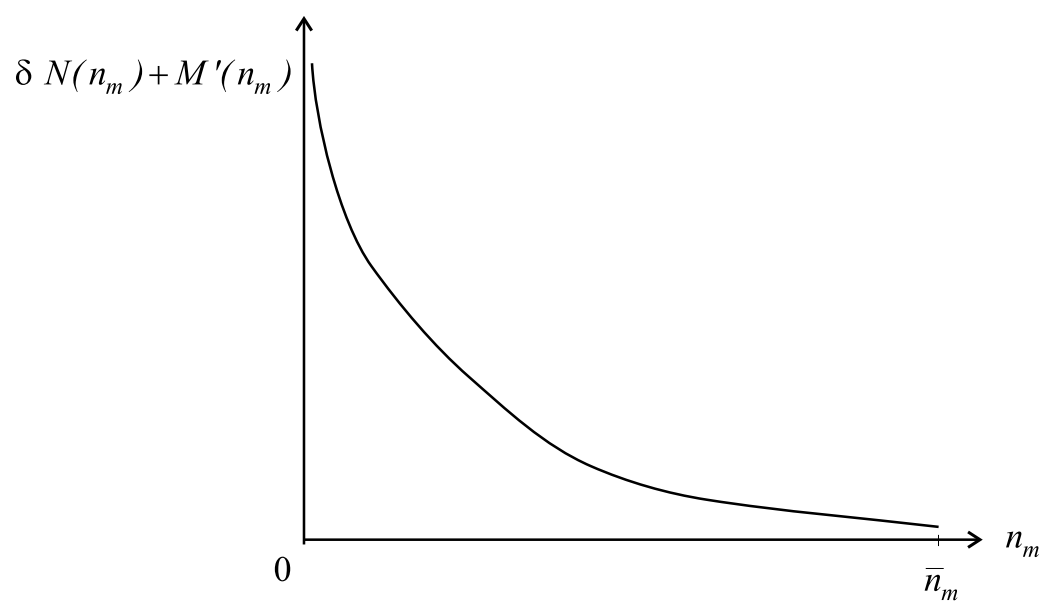

Figure 1: The numerical example $\pi_{g} / \pi_{s}=0.00001$

Note, however, that the farmer will not become active unless the present value of her profits along the optimal path is nonnegative. In the polar case $\pi_{g}=0, \pi_{f}>0$ and $\pi_{s}>0$ (hence $\pi_{g} / \pi_{s}=0$ ) the farmer's instantaneous profits, $\pi_{g}\left(\bar{n}_{g} G\left(f_{t}, n_{m t}\right)-\bar{n}_{g}\right)-\pi_{f} f_{t}-\pi_{s} s_{t}$,

\footnotetext{
${ }^{8}$ Figures 1-3 are freehand drawings emphasizing the main properties of the function $\delta N\left(n_{m}\right)+M^{\prime}\left(n_{m}\right)$ in a stylized way only. The exact plotted graphs can be obtained from the authors upon request.
} 
are obviously negative for all points in time in which she would choose $f_{t}>0$ or $s_{t}>0$. With very large $\pi_{s}$ and very small $\pi_{g}$ the price ratio $\pi_{g} / \pi_{s}$ is small and can be made arbitrarily close to zero. But when $\pi_{g} / \pi_{s}$ approaches zero, there is some small positive threshold value of $\pi_{g} / \pi_{s}$, say $\left(\pi_{g} / \pi_{s}\right)^{*}$ such that present value profits are negative if and only if $\pi_{g} / \pi_{s}$ is less than $\left(\pi_{g} / \pi_{s}\right)^{*}$. To demonstrate that Case I is not void we set $\pi_{g} / \pi_{s}=0.00001$ in a numerical example and calculated the associated present-value profit as 0.00049 .

Case II: If $\pi_{g} / \pi_{s}$ is neither too small nor too large, it may be optimal to switch from zero pesticides or maximum pesticides to an intermediate application of pesticides.

For a certain range of values of $\pi_{g} / \pi_{s}$, the $\delta N\left(n_{m}\right)+M^{\prime}\left(n_{m}\right)$ curve exhibits two points of intersection with the $n_{m}$-axis as illustrated in Figure 2 for $\pi_{g} / \pi_{s}=0.01$. In that case part (ii) of Proposition 2 applies according to which either (a) the FPAP to the first intersection point $\hat{n}_{m 1}$ or (b) the FPAP to the maximum population $\bar{n}_{m}=N^{m}(0)$ is optimal.

In case II(a) the optimal pest control path crucially depends on the initial mice population $n_{m 0}$. Suppose first $n_{m 0}<\hat{n}_{m 1}$. It is then optimal to set $s_{t}=0$ during some initial time interval as long as $n_{m t}<\hat{n}_{m 1}$. Under that policy the mice population will grow until eventually, say at $t=\bar{t}, n_{m \bar{t}}=\hat{n}_{m 1}$ is reached. Then it is optimal to switch from $s=0$ to some suitable $s \in] 0,1 / c_{m}[$ for all $t>\bar{t}$.

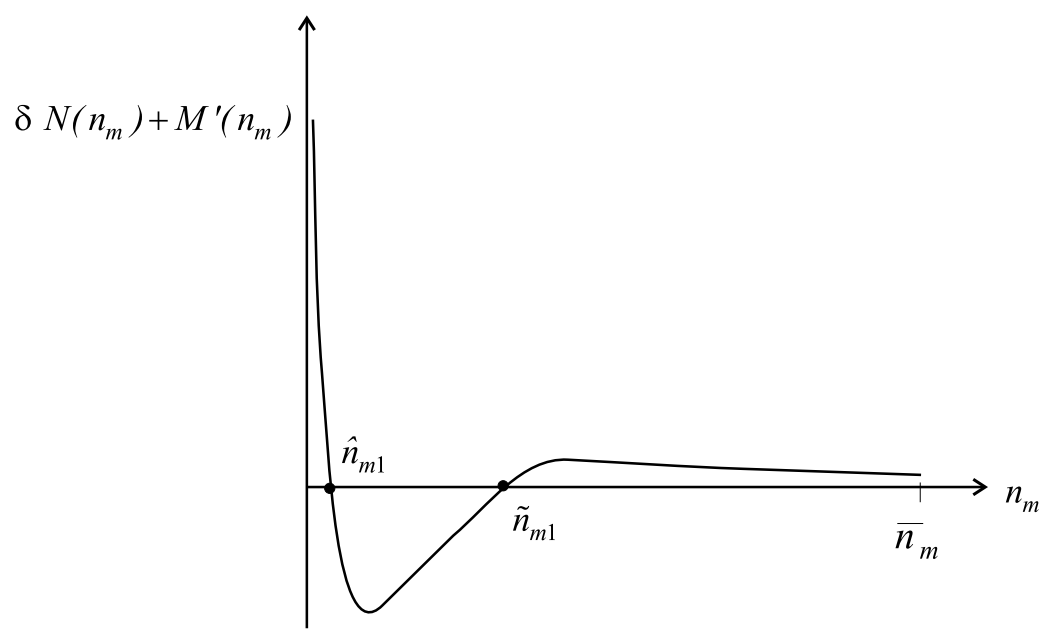

Figure 2: The numerical example $\pi_{g} / \pi_{s}=0.01$

Suppose now, $n_{m 0}>\hat{n}_{m 1}$. Under this condition it is optimal to set $s_{t}=1 / c_{m}$ during some initial time interval as long as $n_{m t}>\hat{n}_{m 1}$. As a consequence of that policy, the mice population will shrink until eventually, say at $t=\bar{t}, n_{m \bar{t}}=\hat{n}_{m 1}$ is reached. Then it is optimal to switch from $s=1 / c_{m}$ to some suitable $\left.s \in\right] 0,1 / c_{m}[$ for all $t>\bar{t}$.

Consider now the Case II(b) where the FPAP to $\bar{n}_{m}$ is optimal. That scenario is 
obviously identical to Case I described above. As in Case I one needs to take care of the non-negativity condition of present-value profits. ${ }^{9}$

Case III: If $\pi_{g} / \pi_{s}$ is sufficiently large, it is optimal to apply a positive but moderate amount of pesticides along the entire time path.

Figure 3 shows for $\pi_{g} / \pi_{s}=20$ that the $\delta N\left(n_{m}\right)+M^{\prime}\left(n_{m}\right)$ curve intersects the $n_{m}$-axis exactly once at some $\left.\hat{n}_{m 1} \in\right] 0, \bar{n}_{m}$ [. According to part (i) of Proposition 2 for $q=1$ the FPAP to $\hat{n}_{m 1}$ is therefore optimal. The optimal pest control is the same in qualitative terms as the optimal policy in Case II(a) described above.

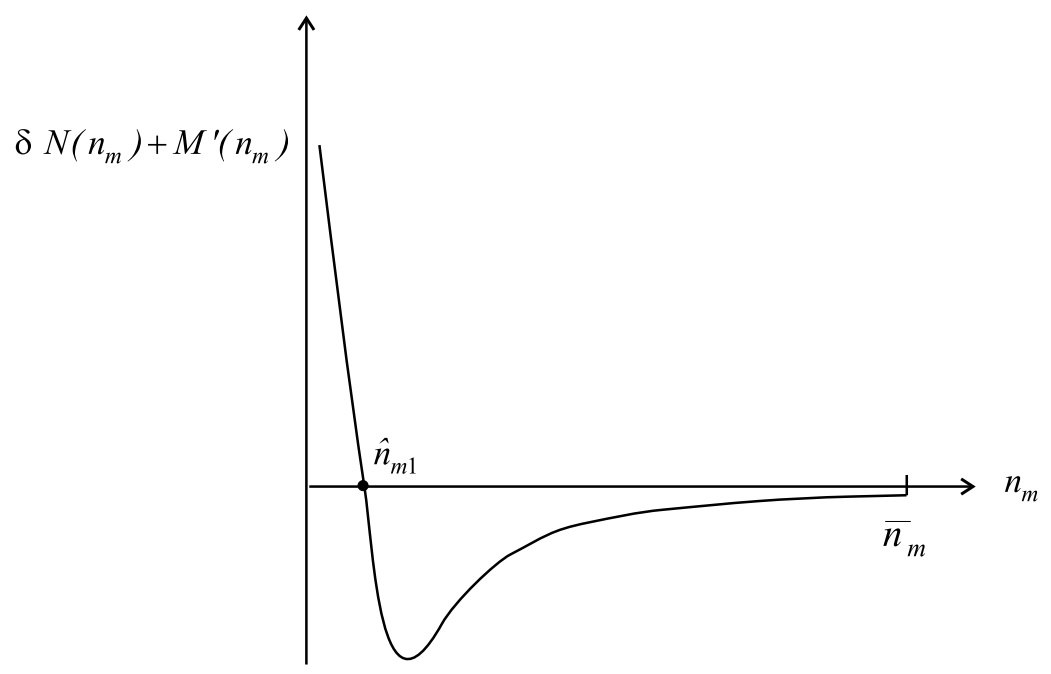

Figure 3: The numerical example $\pi_{g} / \pi_{s}=20$

Convex Problem We now reintroduce the assumption $\mu \in] 0,1[$ to reconsider the original convex optimization problem. Note first that those solutions $\left\{s_{t}\right\}$ of the linearized problems that involve intermediate values $s \in] 0,1 / c_{m}$ [ for some time interval or for the entire time path are no candidates for a solution to the convex problem since such paths $\left\{s_{t}\right\}$ cannot maximize the Hamiltonian of the convex problem. Solution candidates for the convex problem are necessarily boundary solutions, either $s=0$ or $s=1 / c_{m}$. When this observation is combined with the fact that the values of the linearized and the convex problem must coincide in case of boundary solutions we conclude that if a boundary solution is optimal for the linearized problem, it is optimal for the convex problem, too. Thus, whenever $s_{t}=0$ for all $t \geq 0$ is the optimal solution of the linearized problem (the Cases I and II(b) above), it is also the optimal solution of the convex problem.

\footnotetext{
${ }^{9}$ Two additional special cases should be briefly mentioned. If the initial mice population happens to equal $\hat{n}_{m 1}$ or $\bar{n}_{m}$ (in Figure 2) $\left.s \in\right] 0,1 / c_{m}$ [ or $s=0$ is optimal right from the beginning.
} 
While this result gives us a partial characterization of the solution to the convex problem, we have not yet any information about the solution to the convex problem under parameter constellations in which the linearized problem exhibits an interior solution during some time interval. Progress can be made by applying Theorem 3.4 of Feichtinger and Hartl (1986, p. 79) which reads, adapted to the present context:

Proposition 3. As the optimum value of the convex problem can never coincide with the optimum value of the linearized problem in case of an interior solution, the linear optimum constitutes an upper bound of the convex problem. This upper bound can be approximated arbitrarily closely by choosing a chattering control: the farmer should switch as often as possible from $s=1 / c_{m}$ to $s=0$ observing the condition that the average level of $s$ corresponds to the optimum level of $s$ for the linearized problem.

Chattering pest control consists of alternating time intervals of massive use of pesticide and no use of pesticide at all. Heavy pest control is exercised after the pest population has significantly grown, and it is continued until the pest population is sufficiently decimated. Then an interval follows with no application of pesticides at all, allowing the pest population to recover and grow until eventually a new round of massive pest control is due etc. Casual evidence for this kind of pest control can be found in practice. The interesting feature of Proposition 3 is in this context that conditions are spelled out under which a chattering pest control is optimal. Intuitively speaking, the relevant condition is that the strategy of a two-interval sequence of massive control followed by zero control is less expensive than the alternative strategy of continuous moderate application of pesticide over both intervals provided that both strategies generate the same yield. The economic advantage of the chattering control over a moderate continuous control is shown to depend on the specific way in which pesticides impact on the net offspring of mice and on the functional form of the net offspring function of the representative mouse ( $\mu=1$ versus $\mu \in] 0,1 / c_{m}[)$. It is also worth pointing out that the properties of the instantaneous grain production function with respect to $n_{m}$ as derived in Proposition 1 do not seem to play a role in determining which type of pest control is optimal.

\section{Optimal steady-state pest control}

In this section we assume that the farmer ignores the adjustment path to the steady state and accounts only for steady-state mice populations in her profit-maximizing calculus. This strategy is the better an approximation for problem (18), the faster is the adjustment to the steady state. To explore that case we insert $n_{m}=N^{m}(s)$ from (14) into $\bar{n}_{g} G\left(f, n_{m}\right)-\bar{n}_{g}$ 
and interpret it as the farmers's long-run production function. The farmer now solves for $f$ and $s$ the static Lagrangean

$$
\mathcal{L}=\pi_{g}\left[\bar{n}_{g} G\left(f, N^{m}(s)\right)-\bar{n}_{g}\right]-\pi_{f} f-\pi_{s} s+\rho\left(1-c_{m} s\right) .
$$

\section{Proposition 4 .}

(i) There is a choke price $\left(\pi_{s} / \pi_{g}\right)_{o}>0$ of pesticide in terms of grain such that it is optimal to apply no pesticide, if and only if $\left(\pi_{s} / \pi_{g}\right)>\left(\pi_{s} / \pi_{g}\right)_{o}$.

(ii) If $\left(\pi_{s} / \pi_{g}\right)<\left(\pi_{s} / \pi_{g}\right)_{o}$ and $\theta_{g}>\hat{\theta}_{g}$ with $\hat{\theta}_{g}$ as defined in Proposition 1(ii)(b), the optimal pest control is $s=1 / c_{m}$ implying the eradication of the entire mice population.

(iii) If $\left(\pi_{s} / \pi_{g}\right)<\left(\pi_{s} / \pi_{g}\right)_{o}$ and $\theta_{g}<\hat{\theta}_{g}, s=1 / c_{m}$ (eradication) is also optimal for sufficiently small $\pi_{s} / \pi_{g}$; otherwise a moderate use of pesticide is optimal securing the mice's survival.

Proof: Central to the proof of Proposition 4 is the curvature of $G\left(f, N^{m}(s)\right)$ with respect to $s$. Combining the derivatives

$$
\frac{\mathrm{d} g}{\mathrm{~d} s}=G_{n_{m}} N_{s}^{m} \quad \text { and } \quad \frac{\mathrm{d}^{2} g}{\mathrm{~d} s^{2}}=G_{n_{m} n_{m}} N_{s}^{m}+G_{n_{m}} N_{s s}^{m}
$$

with (14) and Proposition 1 we find that

(i) $G\left(f, N^{m}(s)\right)$ is strictly increasing in $s$, and

(ii) $G\left(f, N^{m}(s)\right)$ is strictly concave (convex) in $s$ if and only if $\theta_{g}<\hat{\theta}_{g}\left(\theta_{g}>\hat{\theta}_{g}\right)$.

The rest of the proof of Proposition 4 is straightforward. ${ }^{10}$

Comparing the optimal dynamic pest control and the optimal steady-state pest control exhibits remarkable differences. In dynamic control mice eradication is never optimal. In contrast, steady state control eradication is always optimal unless (i) $\theta_{g}<\hat{\theta}_{g}$ and (ii) $\pi_{s} / \pi_{g}$ is moderate but doesn't exceed $\left(\pi_{s} / \pi_{g}\right)_{o}$. Surprisingly, the size of the micro parameter $\theta_{g}$ specifying feeding characteristics of grain in the presence of predation turns out to have an impact on optimal steady state farming while that parameter does not influence the qualitative properties of the optimal dynamic pest control.

\footnotetext{
${ }^{10}$ Note that the first order condition of $(25)$ may characterize a local minimum or a local maximum of profit while the global maximum is attained as a corner solution where mice are eradicated.
} 


\section{Summary and extensions}

This paper provides both a microfoundation of predator-prey population growth which depends on pesticides and fertilizers and a microfoundation of the agricultural production function. It turns out that the production function may be either concave or convex depending on species micro parameters. A convex function makes it optimal to kill the entire pest population when the farmer restricts her attention to steady states. Solving the full dynamic optimal pest control problem, the farmer either applies no pesticides at all or uses the chattering pest control which requires to switch as often as possible between no pesticides and the maximum amount of pesticides.

There are several extensions of our model which are worth mentioning. First, one could add a third species, say buzzards that feed on mice. In such an extended model an interesting issue would be to compare conventional farming, i.e. the use of fertilizer and pesticide, and green farming, e.g. improving the living conditions of buzzards (Pethig 2004). Another promising extension would be an analysis of indirect effects of pesticides. If the resource is water, pesticides may contaminate that water and impair the growth of grain. Another impact of pesticides could consist in a reduction of grain quality. The grain price, $\pi_{g}$, may be positively correlated with quality (hedonic price function) to the effect that the grain price falls with the quality of grain deteriorating after the use of pesticides. When the farmer faces shrinking revenues from selling grain it might be profitable for her to use less pesticide. Such a tradeoff between falling sales prices and increasing the quantity of grain (of decreasing quality) through pesticides tends to reduce the application of pesticides. This effect is capable to foster organic farming provided the quality-price link does materialize in the market place.

\section{References}

Babcock B. A., Lichtenberg E. and D. Zilberman (1992), Impact of damage control and quality of output: Estimating pest control effectiveness, American Journal of Agricultural Economics 74, 163-172.

Berck P. and C. Helfand (1990), Reconciling the von Liebig and differentiable crop production functions, American Journal of Agricultural Economics 72, 985-996.

Carrasco-Tauber C. and L. J. Moffitt (1992), Damage control econometrics: Functional specification and pesticide productivity, American Journal of Agricultural Economics 74, 158-162. 
Chambers R. G. and E. Lichtenberg (1996), A non-parametric approach to the von LiebigParis technology, American Journal of Agricultural Economics 78, 373-386.

Christiaans T., Eichner T. and R. Pethig (2005), A micro-level 'consumer approach' to species population Natural Resource Modeling, in press.

Crocker T. D. and J. Tschirhart (1992), Ecosystems, externalities and economies, Environmental and Resource Economics 2, 551-567.

Eichner T. and R. Pethig (2003), A microfoundation of predator-prey dynamics, Discussion Paper 110-03, University of Siegen.

Eichner T. and R. Pethig (2004), Economic land use, ecosystem services and microfounded species dynamics, Discussion Paper, University of Siegen.

Feder G. and U. Regev (1975), Biological interactions and environmental effects in the economics of pest control, Journal of Environmental Economics and Management 2, 75-91.

Feichtinger G. and R. F. Hartl (1986), Optimale Kontrolle ökonomischer Prozesse, de Gruyter, New York.

Finnoff D. and J. Tschirhart (2003a), Protecting an endangered species while harvesting its prey in a general equilibrium ecosystem model, Land Economics 79, 160-180.

Finnoff D. and J. Tschirhart (2003b), Harvesting in an eight-species ecosystem, Journal of Environmental Economics and Management 45, 589-611.

Hall D. C. and R. B. Norgaard (1973), On the timing and application of pesticides, American Journal of Agricultural Economics 55, 198-201.

Hannon B. (1976), Marginal product pricing in the ecosystem, Journal of Theoretical Biology 56, 253-267.

Pethig R. (2004), Agriculture, pesticides and the ecosystem, Agricultural Economics 31, $17-32$.

Pethig R. and J. Tschirhart (2001), Microfoundations of population dynamics, Journal of Bioeconomics 3, 27-49.

Tschirhart J. (2000), General equilibrium of an ecosystem, Journal of Theoretical Biology 203, 13-32.

Tschirhart J. (2002), Resource competition among plants: From optimizing to community structure, Ecological Modelling 148, 191-212. 
Tschirhart J. (2004), A new adaptive system approach to predator-prey modeling, Ecological Modelling 176, 255-276. 